\title{
Chair rise strategies in older adults with functional limitations
}

\author{
Donna Moxley Scarborough, MS, PT; ${ }^{1-2 *}$ Chris A. McGibbon, PhD; ${ }^{1-3}$ David E. Krebs, DPT, PhD ${ }^{1-2}$ \\ ${ }^{1}$ Department of Orthopedics, Biomotion Laboratory, Massachusetts General Hospital (MGH), Boston, MA; ${ }^{2}$ MGH \\ Institute of Health Professions, Boston, MA; ${ }^{3}$ Institute of Biomedical Engineering, University of New Brunswick, \\ Fredericton, NB, Canada
}

\begin{abstract}
Studies investigating chair rise (CR) strategies in older adults, including the identification of CR strategies in the clinical setting, are limited. We identified biomechanical differences between CR strategies performed by older adults. The "healthy" momentum transfer (MT), the exaggerated trunk flexion (ETF), and the dominant vertical rise (DVR) CR strategies were observed in 29 women and 17 men (64-88 yr) with functional limitations. The DVR strategy required the greatest knee torque (mean $=12.76$ moment-\% body weight). Maximum knee torque occurred significantly earlier for the ETF strategy (mean $=47 \%$ CR time). Lift-off time was earliest for the ETF strategy (mean $=32 \%$ CR time). Peak trunk flexion was the primary distinguishing biomechanical measure for classifying CR strategy. This finding may offer clinicians an easy method of identifying CR strategies during evaluation. Because of DVR and ETF movement timing and torque demands, we conclude that MT is the safest and most preferable CR strategy.
\end{abstract}

Key words: chair rise, dominant vertical rise, exaggerated trunk flexion, functional limitations, momentum transfer, movement strategy, older adults, peak trunk flexion, rehabilitation, sit-to-stand.

\section{INTRODUCTION}

The ability to rise safely from a chair is a key component of functional mobility. However, many older adults have difficulty rising from a chair successfully [1-2]. Tinetti et al. reported a high occurrence of falls during sit-to-stand among older adults [1]. Safe transition from static sitting to upright standing requires adequate motor control and dynamic stability. Upper-body movement, lower-limb strength, and coordination of segment momentum generation not only contribute to the success of sit-to-stand but also define the movement strategy used to attain the standing position [3-11]. Most clinicians focus primarily on the chair rise (CR) success or failure outcome. Focus on the process of attaining the upright position has been limited. Clinicians could improve treatment plans for CR mobility among older adults if, based on identifying an observed movement strategy, they could determine which components of motor control are likely impaired. This study identifies the differences between CR strategies of older adults with functional limitations.

Abbreviations: $3-\mathrm{D}=$ three-dimensional, $\mathrm{A}-\mathrm{P}=$ anteroposterior, $\mathrm{COG}=$ center of gravity, $\mathrm{CR}=$ chair rise, $\mathrm{DVR}=$ dominant vertical rise, ETF = exaggerated trunk flexion, LED = light-emitting diode, MGH = Massachusetts General Hospital, $\mathrm{MT}=$ momentum transfer.

* Address all correspondence to Donna Moxley Scarborough, MS, PT, Clinical Research Project Manager/ Research Physical Therapist; Harris Orthopedic Biomechanics and Biomaterials Laboratory, Massachusetts General Hospital, 55 Fruit Street, GRJ 1125, Boston, MA 02114; 617-724-5103; fax: 617-726-3883.

Email: dscarborough@partners.org

DOI: 10.1682/JRRD.2005.08.0134 
CR in nondisabled younger and older adults has been studied during unconstrained conditions, where arm movement for assistance and free positioning on the seat are allowed [6,10,12], as well as during various constrained CR conditions [3-5,7,11,13-15]. These studies suggest that during the momentum transfer (MT) CR strategy, the upper-body transfers forward momentum to vertical momentum and continued forward momentum when the person lifts the buttocks off the chair ("lift off") [3-4]. The MT strategy appears as a smooth movement, with simultaneous back and knee extension after lift off [7]. Schenkman et al. and Hughes and Schenkman described another CR strategy, the stabilization strategy, used by older adults with functional limitations, in which they flex the trunk to place the center of mass over the feet before lifting off the seat. In this strategy, lift off from the seat is accomplished without assistance from vertical momentum [3-4]. This strategy is also referred to as the exaggerated trunk flexion (ETF) strategy, based on the excessive trunk flexion that is observed during the majority of the CR [7]. The dominant vertical rise (DVR) strategy involves limited trunk motion and rising is predominantly in the vertical direction [7]. The MT strategy, however, has generally been recognized as the ideal and most efficient strategy performed by nondisabled persons of all ages [3-5,7,13-14].

Our objectives were to characterize the biomechanical differences between CR strategies. To our knowledge, how the ETF and the DVR CR strategies differ from the MT strategy has not been analyzed biomechanically. Based on previous studies, we developed and expanded upon several hypotheses [3-5,7,11]: (1) biomechanical factors discriminate CR strategies; (2) the ratio of peak upper-body anterior momentum to peak upper-body vertical momentum will be closest to 1.0 for persons in the MT strategy group; (3) because of the apparent efficient use of momentum and the observed simultaneous knee and back extension during the MT strategy, people in the MT strategy group will demonstrate smaller knee-torque values than people using DVR or ETF strategies; and (4) people using the ETF strategy will take longer to complete CR than people using the MT strategy. Understanding how CR strategies differ will provide the basis for investigating why people may use one strategy versus another. Ultimately, this knowledge of older adults' CR strategies may lead to improved treatment recommendations for functional training of the CR activity.

\section{METHODS}

\section{Subjects}

We screened 95 community-dwelling older adults with self-reported functional limitations who were participants in a home-based strengthening study. Detailed biomechanical CR analysis was conducted with the chair height set at 100 percent of each individual's knee height [16-17]. Subjects were classified as having a functional limitation and included if they reported limitations on one or more of the nine physical functioning items (excluding the vigorous activity item) on the 36-item Short Form [16-17]. Subjects were excluded if they had a diagnosis of active terminal disease or progressive disease with fluctuating symptoms that could have impeded their safety or ability to consistently participate in the study (Table 1). Each subject determined which diagnosis was the primary cause for his or her current functional limitations. This primary diagnosis is reported for all subjects. Each subject agreed to participate in the study and signed a written consent. This research was approved by the Massachusetts General Hospital (MGH) Institutional Review Board.

\section{Biomechanical Testing}

Kinematic and kinetic data acquisition is described in detail elsewhere [18-21]. The instrumentation included two Kistler piezoelectric force plates (Kistler Instruments, Winterthur, Switzerland), four Selspot $\mathrm{II}^{\mathrm{TM}}$ optoelectronic cameras (Selective Electronics, Partille, Sweden), and 64 infrared light-emitting diodes (LEDs). Three to five infrared LEDs, embedded in rigid plastic discs, were securely attached to 11 body segments (right and left feet, shanks, thighs, and arms; and the pelvis, trunk, and head), and 6 degree-of-freedom kinematics of the 11 body segments, with resolution of $<1^{\circ}$ and $<1 \mathrm{~mm}$, were generated [20]. A three-dimensional (3-D) android model of the subject as well as calculations of the dependent variables were generated by the SuperTrack software developed at the Biomotion Laboratory, MGH, Boston, Massachusetts.

A backless, armless, firm chair was adjusted for each individual to the height of the medial tibial plateau (knee height). Each subject was seated with the greater trochanters $4 \mathrm{~cm}$ from the seat's front edge with ankles in $18^{\circ}$ dorsiflexion and bare feet $10 \mathrm{~cm}$ apart [7]. The instructions given to each subject were "Rise up from the chair as you normally would; once standing, stand as still 
Table 1.

Subject diagnosis and functional limitation characteristics.

\begin{tabular}{|c|c|c|c|}
\hline Variable & $\begin{array}{l}\text { Momentum Transfer } \\
\qquad(n=16)\end{array}$ & $\begin{array}{l}\text { Exaggerated Trunk Flexion } \\
\qquad(n=16)\end{array}$ & $\begin{array}{l}\text { Dominant Vertical Rise } \\
\qquad(n=14)\end{array}$ \\
\hline Primary Diagnosis & $\begin{array}{l}\text { Angina, asthma, CAD, COPD, } \\
\text { diffuse arthritis, emphysema, } \\
\text { general deconditioning, herni- } \\
\text { ated disk, HX lung cancer (TX } \\
\text { completed, in remission), HX } \\
\text { polio, low back pain, sciatica, } \\
\text { total knee replacement. }\end{array}$ & $\begin{array}{l}\text { Asthma, diabetes, diffuse } \\
\text { arthritis, diffuse weakness, } \\
\text { general deconditioning, herni- } \\
\text { ated disk, right foot drop, sci- } \\
\text { atica, shortness of breath, } \\
\text { stroke. }\end{array}$ & $\begin{array}{l}\text { Angina, COPD, diffuse arthri- } \\
\text { tis, general deconditioning, } \\
\text { gout, herniated disk, HX lung } \\
\text { cancer (TX completed, in remi- } \\
\text { ssion), low back pain, osteo- } \\
\text { arthritis hips/knees, pseudo- } \\
\text { gout, rotator cuff injury. }\end{array}$ \\
\hline \multicolumn{4}{|c|}{ Diagnosis Classification $^{*}(n)$} \\
\hline Cardiopulmonary & 7 & 2 & 3 \\
\hline Neuromuscular & 1 & 2 & 0 \\
\hline Musculoskeletal & 8 & 12 & 9 \\
\hline Systemic Conditioning & 0 & 0 & 2 \\
\hline \multicolumn{4}{|l|}{ Functional Limitation $^{\dagger}$} \\
\hline Mean & 7.4 & 5.3 & 5.5 \\
\hline Mode & 11 & 4 & 2 \\
\hline Minimum & 1 & 1 & 2 \\
\hline Maximum & 11 & 14 & 15 \\
\hline
\end{tabular}

as possible until told to relax.” Each subject performed one practice trial and two test trials. CR trials were considered successful when the subjects maintained the requested foot position and their arms remained folded throughout the 7-second data collection trial without requiring assistance from the tester. The second CR test trial was used for data analysis. The CR time was calculated as the time from start of movement through end of rise. The time at which forward upper-body momentum began, i.e., became $>0$, defined the start of movement $[7,11]$. End of rise was defined as the time at which the whole body center of gravity (COG) reached its highest vertical position [7]. In addition, we defined the time of lift off from the chair as the time at which the thigh segment moved $2^{\circ}$ upward from its original position [7]. We analyzed maximum trunk flexion (relative to room coordinates), kinetic energy $\left(1 / 2 M V^{2}\right.$, where $M=$ [constant] mass and $V=$ COG translational velocity resultant of all 3 dimensions) at the time of lift off, and maximum hip and knee torques [18] between lift off and end of rise. The timing in percent CR time of lift off, maximum upperbody vertical and anteroposterior (A-P) linear momentum, maximum kinetic energy, maximum trunk flexion, and maximum hip and knee torque values (normalized to body weight) were also compared across the three strategies.

\section{Chair Rise Strategy Classification}

The three CR strategies were defined based on the observation of the 3-D android representation of each subject during CR (Figure 1) [7]. One investigator (with $>15$ years experience) classified all the subjects' CR strategies based on the following definitions [7]. The MT strategy is defined as upper-body flexion during lift off and continuing through the initiation of knee extension, with smooth transition to simultaneous back and knee extension [7]. The ETF strategy, also referred to as the stabilization strategy [3-4], is defined as ETF prior to lift off, frequently followed by further trunk flexion. This excessive trunk flexion places the COG over the feet during lift off, which results in delayed trunk extension during final transition to the erect posture [7]. The DVR strategy is defined as cessation of forward trunk flexion immediately at lift off and followed by dominant vertical COG displacement and knee-hip extension [7], with trunk extension movement delayed until after knee-hip extension is completed. Based on these CR classifications [7], 

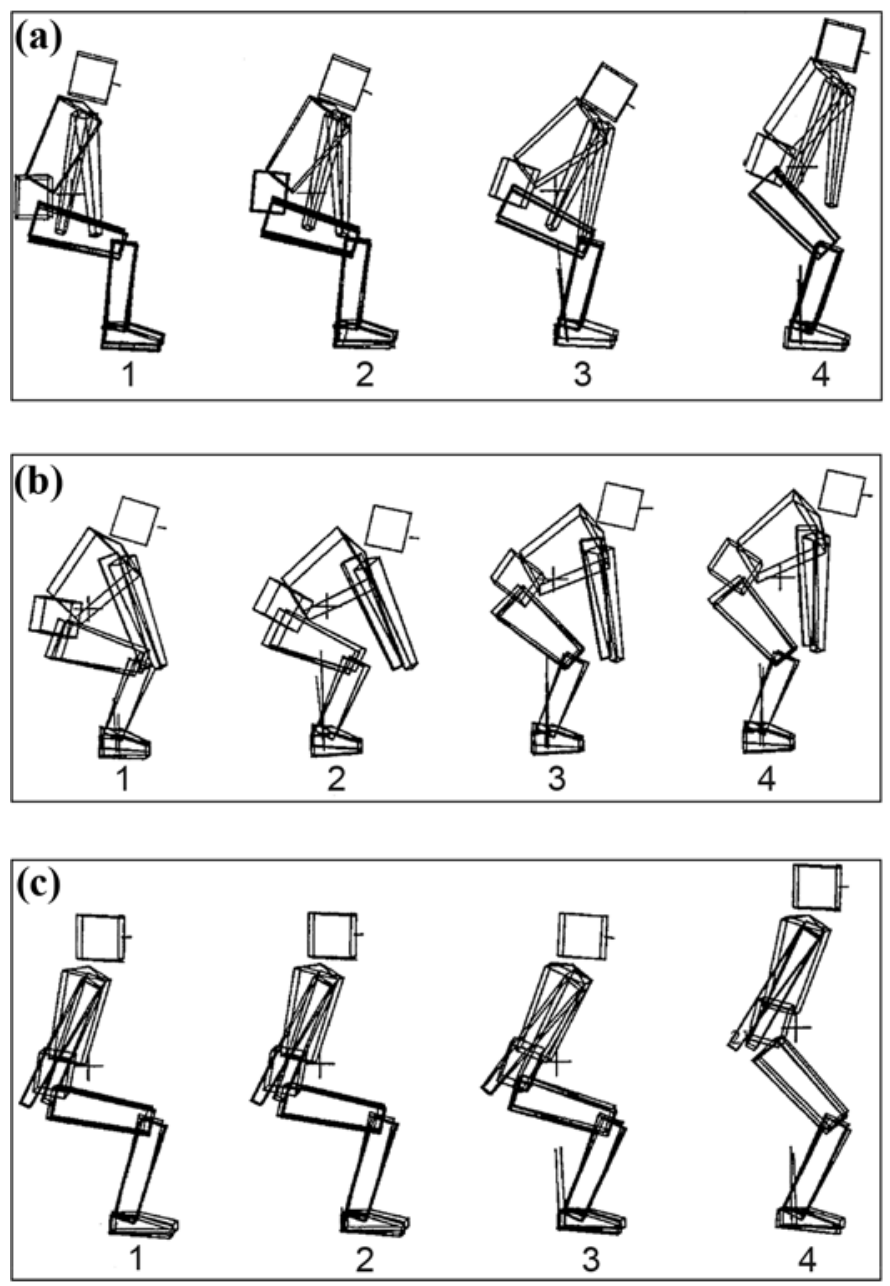

Figure 1.

Android models from representative individual subjects for (a) momentum transfer (b) exaggerated trunk flexion, and (c) dominant vertical rise chair-rise strategies. Time events represented as follows: $1=$ lift off, 2 = maximum anteroposterior linear momentum, 3 = maximum trunk flexion, 4 = maximum vertical linear momentum. Vertical line originating from force plates within floor represents ground reaction force. Cross at pelvis represents whole body center of gravity.

we discovered that most of the subjects used the MT strategy. Of the 95 subjects able to independently perform CR at 100 percent knee height, 14 subjects performed the DVR strategy and 16 subjects the ETF strategy. Therefore, we used a consecutive sample, by order of visit to the laboratory, of the first 16 subjects who performed the MT strategy to form a similar group size, which resulted in a total sample of 46 subjects (Table 2).

To determine if clusters based on biomechanical data could be identified, we performed a K-means cluster analysis of the following biomechanical CR measures: knee-extension torque, hip-extension torque, and trunk flexion. Then we correlated the clusters with the observed strategies and the best cluster was defined as that which best correlated (Spearman's rank-order correlation coefficient) with the observed strategy group.

We then used a one-way analysis of variance to compare the biomechanical variables across the groups based on the cluster analysis, $\alpha<0.05$. We used post hoc Bonferroni tests, where appropriate, to investigate differences across strategy groups. A chi-square crosstabs analysis based on diagnosis group versus strategy group was performed. We used a Kruskal-Wallis analysis to compare the functional limitation scores across the three CR strategy groups. Statistical Package for the Social Sciences, version 8 (SPSS, Inc, Chicago, Illinois) was used for all analyses.

\section{RESULTS}

No difference in age existed between the three strategy groups (Table 2). The approximate 2:1 ratio of female to male subjects in this study was distributed evenly across the three strategy groups (Table 2). Subject height, weight, and body mass index values were not different between the three strategy groups (Table 2). No significant association between diagnosis and strategy group existed ( $p=0.25$ ). No differences were noted across the three CR strategy groups for self-reported functional limitations scores $(p=0.13)$.

Maximum trunk flexion (relative to the room) was different across strategy groups; peak trunk flexion was greatest for the ETF group followed by the MT group and then the DVR group (Table 3). Trunk flexion was the key variable that resulted in the most consistent cluster analysis grouping relative to the observed strategy groups. The trunk-flexion values for CR strategy classifications based on the cluster analysis can be found in Table 4. Spearman's rank-order correlation coefficient between original CR strategy groupings and the trunk flexion CR groupings from the cluster analysis was notable $\left(r_{s}=0.41, p=\right.$ 0.004).

Torque and momentum data also differed between the CR strategies. Maximum knee-torque values differed significantly across the three CR strategy groups ( $p=$ 0.003) (Figure 2). The average maximum knee torque was lower for the ETF group than the MT group, followed 
Table 2.

Subject characteristics $(N=46)$ according to chair rise strategy (mean \pm standard deviation unless otherwise noted).

\begin{tabular}{lcccc}
\hline \multicolumn{1}{c}{ Variable } & Momentum Transfer & $\begin{array}{c}\text { Exaggerated Trunk } \\
\text { Flexion }\end{array}$ & $\begin{array}{c}\text { Dominant Vertical } \\
\text { Rise }\end{array}$ & Total \\
\hline Age $(\mathrm{yr})$ & & $74.02 \pm 6.66$ & $75.59 \pm 5.62$ & $74.95 \pm 6.50$ \\
Height $(\mathrm{m})$ & $75.34 \pm 7.32$ & $1.69 \pm 0.15$ & $1.65 \pm 0.10$ & $1.65 \pm 0.12$ \\
Weight $(\mathrm{kg})$ & $1.61 \pm 0.09$ & $71.62 \pm 13.88$ & $71.53 \pm 7.85$ & $70.58 \pm 13.41$ \\
Body Mass Index & $68.72 \pm 16.97$ & $26.37 \pm 4.81$ & $26.28 \pm 2.95$ & $26.35 \pm 4.43$ \\
Sex $(n)$ & $26.39 \pm 5.31$ & 11 & & \\
Female & & 5 & 6 & 29 \\
Male & 10 & 5 & 6 & 17 \\
\hline \hline
\end{tabular}

Table 3.

Comparison of kinetics and kinematics and their timing within chair rise (CR) time and across CR strategies (mean \pm standard deviation).

\begin{tabular}{|c|c|c|c|}
\hline Variable & Momentum Transfer & $\begin{array}{c}\text { Exaggerated Trunk } \\
\text { Flexion }\end{array}$ & $\begin{array}{c}\text { Dominant Vertical } \\
\text { Rise }\end{array}$ \\
\hline Max Trunk Flexion* $\left.{ }^{*}{ }^{\circ}\right)$ & $45.71 \pm 5.78$ & $58.11 \pm 7.86$ & $34.88 \pm 7.36$ \\
\hline KE at Lift Off (J) & $3.79 \pm 2.28$ & $4.80 \pm 3.60$ & $4.72 \pm 1.88$ \\
\hline Max Vertical Linear Momentum [(kg/s)/kg - BW] & $0.35 \pm 0.17$ & $0.33 \pm 0.20$ & $0.28 \pm 0.005$ \\
\hline Ratio Max A-P:Max Vertical Linear Momentum & $1.17 \pm 0.23$ & $1.46 \pm 0.45$ & $1.36 \pm 0.30$ \\
\hline CR Time Max $\mathrm{KE}^{\dagger}(\%)$ & $66.44 \pm 13.41$ & $46.59 \pm 19.13$ & $61.12 \pm 18.60$ \\
\hline CR Time Max A-P Linear Momentum (\%) & $43.77 \pm 6.09$ & $37.85 \pm 9.82$ & $43.09 \pm 9.18$ \\
\hline CR Time Max Vertical Linear Momentum (\%) & $72.46 \pm 8.67$ & $65.22 \pm 9.54$ & $71.06 \pm 10.49$ \\
\hline
\end{tabular}

${ }^{*}$ Analysis of variance across strategies, $p<0.001$.

${ }^{\dagger}$ Analysis of variance across strategies, $p<0.05$.

$\mathrm{A}-\mathrm{P}=$ anteroposterior, $\mathrm{BW}=$ body weight, $\mathrm{KE}=$ kinetic energy, Max = maximum.

Table 4.

Cluster analysis results of chair rise strategy groupings, based on degrees of trunk flexion (relative to room).

\begin{tabular}{lccc}
\hline Trunk Flexion ( ${ }^{\circ}$ ) & $\begin{array}{c}\text { Momentum } \\
\text { Transfer }\end{array}$ & $\begin{array}{c}\text { Exaggerated } \\
\text { Trunk Flexion }\end{array}$ & $\begin{array}{c}\text { Dominant } \\
\text { Vertical Rise }\end{array}$ \\
\hline Mean \pm SD & $51.00 \pm 3.76$ & $64.00 \pm 4.57$ & $35.00 \pm 5.37$ \\
Range & $43-57$ & $59-70$ & $24-42$ \\
\hline SD = standard deviation. & & & \\
\hline \hline
\end{tabular}

by the DVR group, with the largest difference between the ETF and DVR groups ( $p=0.003$ ). No difference was noted in average maximum hip torque across strategies $(p=$ 0.07) (Figure 2).

The times at which maximum knee torques occurred were different across the three strategy groups $(p=0.02)$, with the greatest difference between the ETF and DVR strategy groups ( $p=0.04$ ) (Figure 3). However, no differences were noted in the time at which maximum hip torque occurred among strategies. The timing of peak trunk flexion did not differ across strategy groups (Table 3 ).
No differences were found across strategy groups for maximum A-P linear momentum $(p=0.15)$ or vertical linear momentum ( $p=0.51$ ) (Table 3). The difference in the ratio of maximum A-P to maximum vertical linear momentum (maximum A-P:maximum vertical linear momentum) across the three strategy groups was not significant ( $p=0.06$ ) (Table 3). No differences were found across strategy groups in maximum kinetic energy at the time of lift off ( $p=0.95)$ or CR time ( $p=0.08)$ (Figure 4), but the time at which maximum kinetic energy occurred relative to CR time was significantly different $(p=0.008)$ (Table 3). The MT strategy group generated the peak kinetic energy later in the CR than the ETF strategy group $(p=0.007)$. The mean time of lift off as a percent of the CR time was earliest for the ETF strategy group $(p=0.04)$ (Table 3). No difference was noted in the time at which maximum A-P linear momentum occurred across the CR strategy groups ( $p=0.11$ ) or in the time at which maximum vertical linear momentum occurred $(p=0.09)$ (Table 3). 


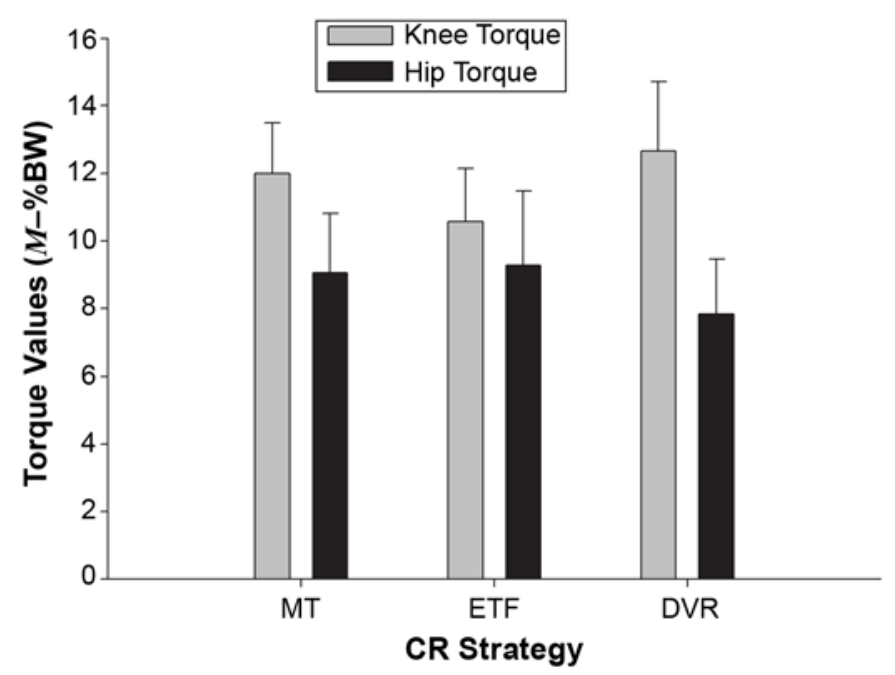

Figure 2.

Average maximum knee and hip torques compared across three strategy groups. Knee torque values were statistically different across three chair rise (CR) strategies, $p=0.003$. Error bars indicate standard deviation. $M=$ moment, $\mathrm{BW}=$ body weight, $\mathrm{MT}=$ momentum transfer, $\mathrm{ETF}=$ exaggerated trunk flexion, DVR = dominant vertical rise.

The people in the ETF strategy group performed CR slower than those in the MT strategy group (Figure 4).

\section{DISCUSSION}

To help older adults who have difficulty rising from a chair, clinicians may offer a variety of instructions including, "move forward, then up," "lean or bend your body forward, bend more, then push up," and "push straight up." These suggestions may or may not result in the desired successful CR, but they do reflect the three observed CR strategies in our study sample. The key biomechanical components we found to identify the three strategies were maximum trunk flexion, maximum knee torque, the time at which maximum kinetic energy occurred, the time of lift off, and the time at which maximum knee torque occurred as percent of CR time. These findings support our hypotheses.

The cluster analysis revealed three distinct groupings of subjects based on the degree of trunk flexion (relative to the room), which correlated with the three original observed CR strategy groups. This result suggests that observing trunk flexion during CR could allow clinicians to identify which CR strategy an individual is performing.

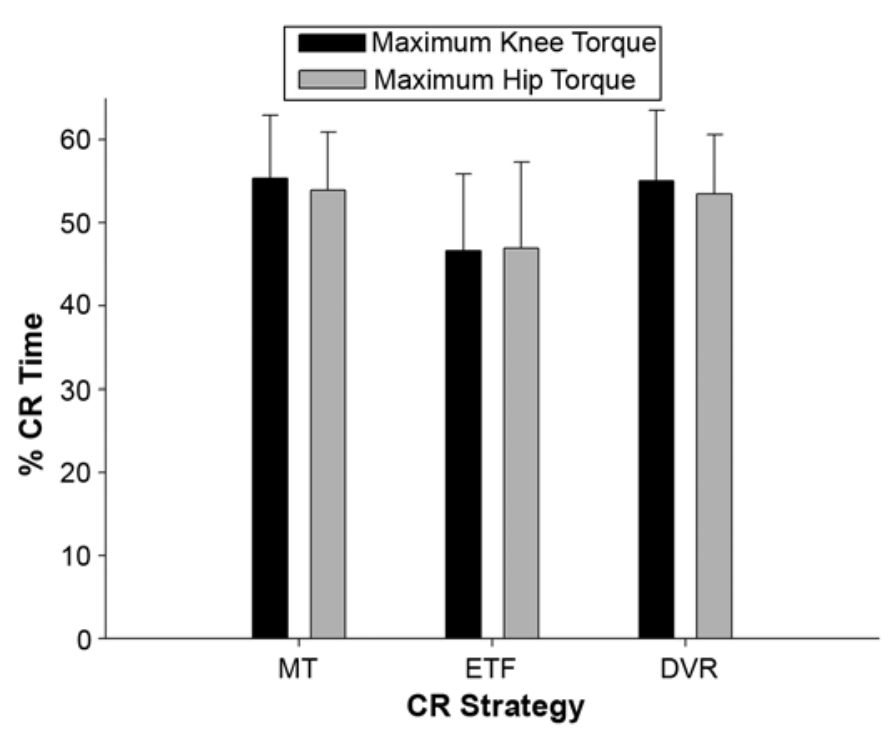

Figure 3.

Timing of maximum knee and hip torque in percent chair rise (CR) time for each strategy. Timing of maximum knee torque was significantly different across three strategies, $p=0.02$. Error bars indicate standard deviation. $\mathrm{MT}=$ momentum transfer, $\mathrm{ETF}=$ exaggerated trunk flexion, DVR $=$ dominant vertical rise.

The MT strategy observed most commonly in nondisabled younger and older adults has been believed to be the most efficient and preferred CR strategy; MT was also most prevalent in our initial sample of older adults [3-4,7]. We hypothesized that the MT strategy group would use anterior and vertical momentum to facilitate a movement pattern that requires lower knee- and hiptorque values and less kinetic energy than the other strategies. The MT strategy group demonstrated simultaneous hip and trunk extension, which resulted in a smooth transitional pattern of movement. Within the MT strategy, the anterior and vertical linear momentum peak magnitude values were similar (Table 3). However, our second hypothesis was not supported: the difference in the ratio of anterior to vertical linear momentum across the three strategies was not statistically significant. Based on our analysis, the MT group appeared to efficiently transfer anterior momentum into vertical momentum, as the descriptive group name implies. The portion of CR after lift off, in which whole-body vertical rise occurs during DVR and ETF strategies, is not facilitated by MT. The ETF and DVR strategies have less anterior momentum of the trunk at the time of lift off, which limits the transfer of anterior to vertical momentum. The point of initial vertical rise is not efficiently fueled during the DVR and 


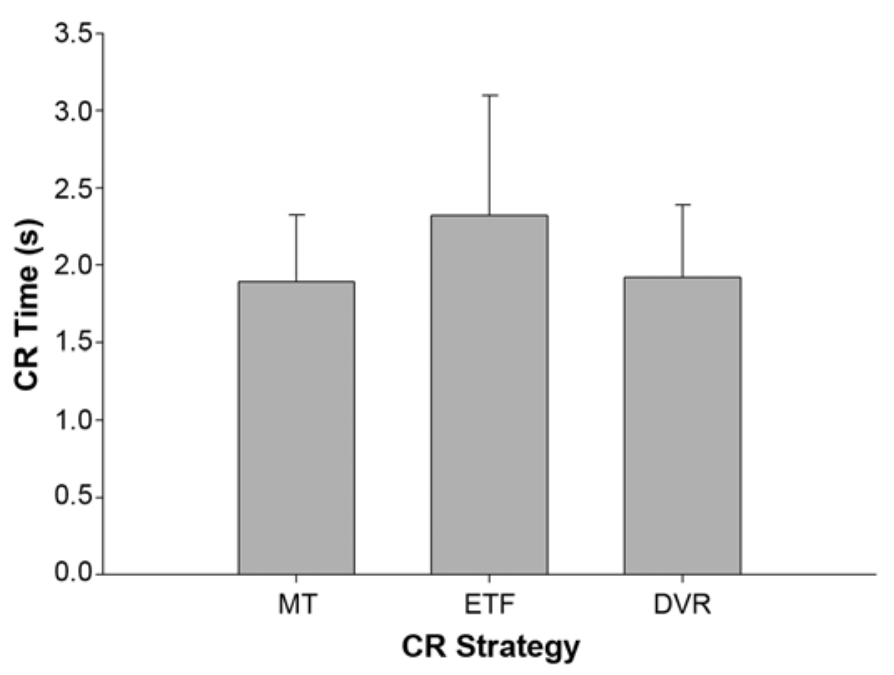

Figure 4.

Average chair rise (CR) time across three strategy groups. Average CR time for momentum transfer (MT) group was significantly faster than that of exaggerated trunk flexion (ETF) group, $p=0.04$. Error bars indicate standard deviation. DVR = dominant vertical rise.

ETF strategies. Therefore, the upward momentum must be generated by the lower-limb and back muscles. The DVR strategy group demonstrated greater knee torque than the ETF group. The MT strategy group generated knee-torque values between the ranges of the DVR and ETF groups. The ETF group used anterior momentum to help attain excessive trunk flexion; however, this limited the vertical momentum generated at lift off. The substantial degree of trunk flexion during the ETF strategy is maintained as the legs perform the vertical rise. Minimalto-absent simultaneous trunk and hip extension exists, which limits the subject's ability to generate anterior to vertical MT. The ETF strategy requires that the backextensor muscles act as primary movers in trunk extension. The DVR group exhibited the greatest knee torques, apparently due to decreased anterior trunk flexion and limited anterior to vertical MT. Thus, an increased reliance exists on lower-limb muscle-force output for performing the DVR CR. These biomechanical findings identify the key factors that create the observable differences in the smooth movement performance of the MT CR strategy versus the other CR strategies.

Our fourth hypothesis was supported; ETF imposes longer CR times than MT. Our analysis included the timing of specific events during CR, in addition to the total CR time. The timing of maximum kinetic energy was different across the three strategies. The ETF group developed maximum kinetic energy earlier than the other strategies and at approximately the same time as peak knee and hip torques. This ETF effect is probably due to the minimal transfer of anterior to vertical momentum and the increased reliance on initial force generated by the lower limbs for the CR. The MT and DVR groups developed peak kinetic energy later in the CR time, after peak trunk flexion and prior to peak vertical momentum. The timing of lift off occurred earlier in the ETF group than the MT group. The ETF group moves the trunk excessively forward to essentially "roll" the buttocks upward, clearing the seat quicker, which results in an earlier lift-off time than the other strategies [11]. As shown in Figure 1, this lifting motion occurs before the active vertical rise. This likely places the person in the transition phase where he or she is not being supported by the chair or in a stable 2-point upright position for longer than the other strategies. Therefore, the ETF strategy, while appearing to provide stability by focusing on moving the COG over the base of support, may actually have a destabilizing effect. As hypothesized, the ETF strategy group performed CR slower than the other strategy groups, probably because of the earlier lift off. The ETF and DVR strategies do not incorporate an optimally coordinated use of trunk and hip-extensor movement or momentum that facilitate CR. Therefore, our data support the theory that both the ETF and the DVR strategies are more physically demanding methods of CR compared with MT.

A previous investigation on failed sit-to-stand trials, when subjects initiate $\mathrm{CR}$ and then sit back down (also referred to as a "sitback") or take a step, provides further insight into CR strategies [11]. Older adults who evinced a sitback or step failure did not generate momentum in the appropriate direction [11]; thus, people using DVR would be more likely than those using MT to encounter a sitback failure, because the DVR group places their COG posterior to the ankle joint throughout the majority of $\mathrm{CR}$ time. Perhaps older adults in the DVR strategy group are fearful that they may fall or become dizzy if they bend their trunk forward and place their head over their feet. The ETF strategy would also likely cause either a sitback failure if at lift off, adequate vertical momentum is not generated or a step failure if anterior momentum is laterally misdirected. Future studies investigating the strategy being performed during a failed CR may support these explanatory hypotheses. 
The similar distribution of age, height, weight, sex, and functional limitation scores cannot explain the variation in CR strategy in our sample of older adults. The amount of functional limitation each subject reported within CR strategy group and the specific type of functional limitation did not explain the differences in strategies performed. Similarly, the distribution of primary diagnosis does not offer an explanation of the performance of different strategies. Prior published data from this study group did not find that quadriceps strength output values correlated with CR strategy performance [7]. The MT CR strategy may be a learned activity. The subject's ability to generate and dampen momentum and move body segments simultaneously during the MT strategy requires that the neuromuscular system provide continuous feedback, which allows for adaptation when needed. Perhaps, degeneration within the neuromuscular control systems accounts for altering movement strategies during CR. The question, "Why do some older adults perform the DVR or ETF CR strategies instead of the MT?” remains for continued investigation. Future investigations of movement patterns may find subjects' leg-muscle force, joint range of motion, visual perception, proprioception, and vestibular input [22] useful in better understanding the variations of movement strategies during well-learned tasks such as CR.

A future study observing older adults performing CR is warranted to validate the use of trunk flexion as a method of classifying CR strategy. The classification of CR strategy was determined by an experienced physical therapist. However, at this time, we have not (nor has anyone to our knowledge) tested the intra- or interrater reliability of observed CR classification. We do suggest this as an important consideration for future studies. Classifying CR strategy with a standardized chair-seat height, starting posture, and foot position, as in this study, may be a useful movement assessment tool for clinicians. Developing physical therapy interventions to help patients implement a desirable CR movement strategy is also highly warranted. Future analysis of CR movement strategies may reveal areas of strengths and deficits in motor control among patients with impairments and functional limitations.

\section{CONCLUSIONS}

Several biomechanical measures discriminate among CR strategies. Peak trunk flexion during CR is the most prominent measure that differentiated the strategies and may be a clinically observable biomechanical measure that could be used to identify CR strategies. Because DVR and ETF strategies impose extra movement timing and torque demands, we conclude that MT is the safest and most preferable CR strategy.

\section{ACKNOWLEDGMENTS}

We acknowledge the cooperation and assistance of the staff at the New England Research Institutes, Watertown, Massachusetts, and the Roybal Center for Enhancement of Late Life Function of Boston University, Strong for Life project, Boston, Massachusetts. We also acknowledge the assistance of the staff at the MGH Biomotion Laboratory, Boston, Massachusetts, and in particular, Jose V. Ramirez, MD; Charmaine Officer, PT, MS; and Dhara Kothari, PT, MS, for assistance in data analysis.

Donna Moxley Scarborough, MS, PT, is former Assistant Clinical Director of the MGH Biomotion Laboratory. Currently, she is a Clinical Research Project Manager and Research Physical Therapist at the Harris Orthopedic Biomechanics and Biomaterials Laboratory, MGH, Boston, Massachusetts.

This material was based on work supported by the National Institutes of Health (grants R01AG12561 and P50AG11669-03) and the Arthritis Foundation.

The authors have declared that no competing interests exist.

\section{REFERENCES}

1. Tinetti ME, Speechley M, Ginter SF. Risk factors for falls among elderly persons living in the community. $\mathrm{N}$ Engl J Med. 1988;319(26):1701-7. [PMID: 3205267]

2. Williamson JD, Fried LP. Characterization of older adults who attribute functional decrements to "old age." J Am Geriatr Soc. 1996;44(12):1429-34. [PMID: 8951311]

3. Schenkman M, Berger RA, Riley PO, Mann RW, Hodge WA. Whole-body movements during rising to standing from sitting. Phys Ther. 1990;70(10):638-48. [PMID: 2217543]

4. Hughes MA, Schenkman ML. Chair rise strategy in the functionally impaired elderly. J Rehabil Res Dev. 1996; 33(4):409-12. [PMID: 8895136$]$

5. Pai Y, Naughton BJ, Chang RW, Rogers MW. Control of body center of mass momentum during sit-to-stand among young and elderly adults. Gait Posture. 1994;2:109-16. 
6. Schultz AB, Alexander NB, Ashton-Miller JA. Biomechanical analyses of rising from a chair. J. Biomech. 1992; 25(12):1383-91. [PMID: 1491016]

7. Scarborough DM, Krebs DE, Harris BA. Quadriceps muscle strength and dynamic stability in elderly persons. Gait Posture. 1999;10(1):10-20. [PMID: 10469937]

8. Kaya BK, Krebs DE, Riley PO. Dynamic stability in elders: Momentum control in locomotor ADL. J Gerontol A Biol Sci Med Sci. 1998;53(2):M126-34. [PMID: 9520919]

9. Millington PJ, Myklebust BM, Shambes GM. Biomechanical analysis of the sit-to-stand motion in elderly persons. Arch Phys Med Rehabil. 1992;73(7):609-17. [PMID: 1622314]

10. Nuzik S, Lamb R, VanSant A, Hirt S. Sit-to-stand movement pattern. A kinematic study. Phys Ther. 1986;66(11): 1708-13. [PMID: 3774881]

11. Riley PO, Krebs DE, Popat RA. Biomechanical analysis of failed sit-to-stand. IEEE Trans Rehabil Eng. 1997;5(4): 353-59. [PMID: 9422460]

12. Alexander NB, Koester DJ, Grunawalt JA. Chair design affects how older adults rise from a chair. J Am Geriatr Soc. 1996;44(4):356-62. [PMID: 8636577]

13. Schenkman M, Hughes MA, Samsa G, Studenski S. The relative importance of strength and balance in chair rise by functionally impaired older individuals. J Am Geriatr Soc. 1996;44(12):1441-46. [PMID: 8951313]

14. Hughes MA, Myers BS, Schenkman ML. The role of strength in rising from a chair in the functionally impaired elderly. J Biomech. 1996;29(12):1509-13. [PMID: 8945648]
15. Papa E, Cappozzo A. Sit-to-stand motor strategies investigated in able-bodied young and elderly subjects. J Biomech. 2000;33(9):1113-22. [PMID: 10854884]

16. Jette AM, Lachman M, Giorgetti MM, Assmann SF, Harris BA, Levenson C, Wernick M, Krebs D. Exercise-It's never too late: The strong-for-life program. Am J Public Health. 1999;89(1):66-72. [PMID: 9987467]

17. Krebs DE, Jette AM, Assmann SF. Moderate exercise improves gait in disabled elders. Arch Phys Med Rehabil. 1998;79(12):1489-95. [PMID: 9862288]

18. Hutchinson EB, Riley PO, Krebs DE. A dynamic analysis of the joint forces and torques during rising from a chair. IEEE Trans Rehabil Eng. 1994;2(2):49-56.

19. Riley PO, Mann RW, Hodge WA. Modeling of the biomechanics of posture and balance. J Biomech. 1990;23(5): 503-6. [PMID: 2373723]

20. Antonsson EK, Mann RW. Automatic 6-D.O.F. kinematic trajectory acquistition and analysis. J Dyn Syst Meas Control. 1989;111(1):31-39.

21. Riley PO, Benda BJ, Gill-Body KM, Krebs DE. Phase plane analysis of stability in quiet standing. J Rehabil Res Dev. 1995;32(3):227-35. [PMID: 8592294]

22. McGibbon CA, Krebs DE, Scarborough DM. Vestibulopathy and age effects on head stability during chair rise. Acta Otolaryngol. 2001;(21(1):52-58. [PMID: 11270495]

Submitted for publication August 8, 2005. Accepted in revised form November 20, 2006. 